\title{
The Design of Interactive Multi-media System for Wireless Environment SUN Danpeng ${ }^{1,}$, JIANG Rui $^{2}$ \\ ${ }^{1,2}$ Qujing Normal University, Qujing 655011, China \\ aSunDanpeng@yeah.net
}

Keywords: multimedia system; wireless environment; interactive

\begin{abstract}
The study on the design of interactive multimedia system is a requirement of the development of information technology and satellite communications. In this paper, the author tries to explore the design of system for wireless environment with RTP agreement for the sake of solving the problems wired network has caused.
\end{abstract}

\section{Background}

The widespread application of computer network, satellite communications increases the humans' reliances on information technology. Moreover, people are more dependent on space information, especially in the outdoor or mobile events. So it is acknowledged that the requirement for the new approaches to get information is urgent. It is apparent that traditional wired networks fail to meet this requirement and then wireless network came into being. Wireless mobile facilities such as PDA, Laptop and wireless network technology such as Bluetooth, wifi, TD-SCHMA offers a mobile access to interactive multi-media function. The multi-media data transmission based on wireless network can be available for the mobile terminal users. Therefore, one good project for the solution to mobile multi-media problems can satisfy the increasing number of demands for the mobile service and enables them to enjoy the shared information without the restriction of time and space. According to this, the design and research on multi-media system for wireless environment has enormous research value and widespread market prospect.

\section{The comparison of several kinds of wireless communication mode}

As the table shows, Wifi adopts DSSS, Direct Sequence Spread Spectrum and CCK technology with the rate up to $53 \mathrm{Mbps}$ and makes internet work flexibly and Independency as the supplementary of wired network. It can be classified into two modes: dot-to-dot mode and fundamental mode. The system in this article selects WIFI wireless communication for two reasons: one is limited experimental circumstances insufficient for the wireless transmission platform; On the other side, 802.11b can transmit more quickly with the speed of $11 \mathrm{Mbps}$ sufficient for the load of huge data transmission

Although the above mentioned wireless communication modes have transfer rates ranging from $1 \mathrm{Mbps}$ to 70Mbps, multimedia audio and video data contains a large amount of information in the process of multimedia network communication and can incorporate tens or hundreds of megabytes of data information without compression. Thus, there is a necessity for compression process in the wireless multi-media network communication aimed at the drawbacks of wireless transmission such as the instability and high error rate.

\section{RTP agreement}

Compared with fixed network, wireless network has the characteristics including instability and high error rate, so RTP agreement can be employed to solve problems such as delay,disorder and incoherent of audio and radio.

Besides, it has many schemes, split connection scheme and end-to-end will be introduced as follows. Split connection scheme divides the source end and CP connection into two segments with fixed $\mathrm{CFH}$ as the source end and $\mathrm{MH}$ as the terminal end with the BS as the connection. One end 
connects FH and BS and the other side connects BS and FH.

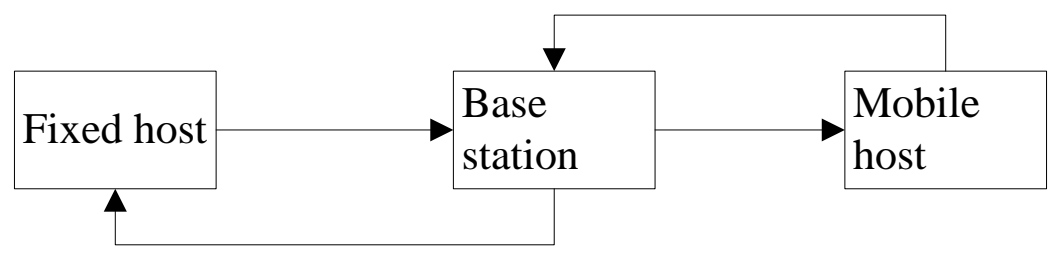

Fig 1 split connection scheme

As can be seen from the diagram, in this end-to-end scheme, only the host on the endpoint is in flow control. Receiving end provides the feedback message and the sending end determines congestion control. The purpose of this scheme is to enable the TCP sender or the receiver can separate congestion of data packets loss and other forms of packet loss. Only when the network congestion occurs, can the TCP congestion control processes be activated, while Other error recovery process for other forms of errors.

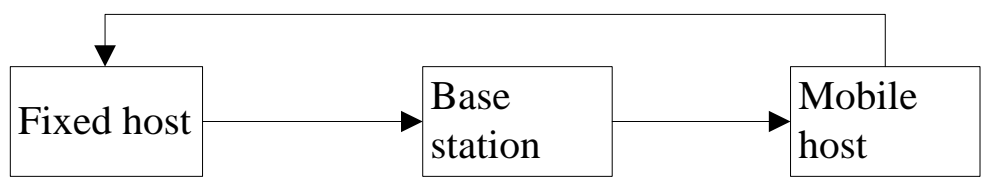

Fig 2 end-to-end scheme

\section{Interactive multimedia design}

\section{Logical Design Sketch Map of Interactive System}

After the design of interactive multimedia system for the wireless environment, we offered logical design of interactive system, as shown in the table, the whole system is designed in accordance with the flow of the inner data with data modules as logical position of each function. The logical design of the whole system is conducted with date and moble chrome as center

\section{RTP Packet}

After RTP Packet is opened, the data generates a new linked node. First compare the time stamp removed with last node time stamp. If it is less than the time stamp of the last node, then compare the time stamp of the penultimate node until time stamp node not greater than that is found.

\section{The comparison of the scheme}

Piece connection scheme is aimed at solving the wireless problem based on the principle of "partial solutions to partial problems" Therefore, wireless and wired environment should be considered respectively to ensure that the cable link remains the same while modify the wireless link protocol. It has hidden the features of TCP wireless link. Besides, both ends of the transmission parameters can be independently optimized, which is also an advantage. But the drawbacks are also salient, which means this scheme violets the conception of end-to-end and increases the burden 


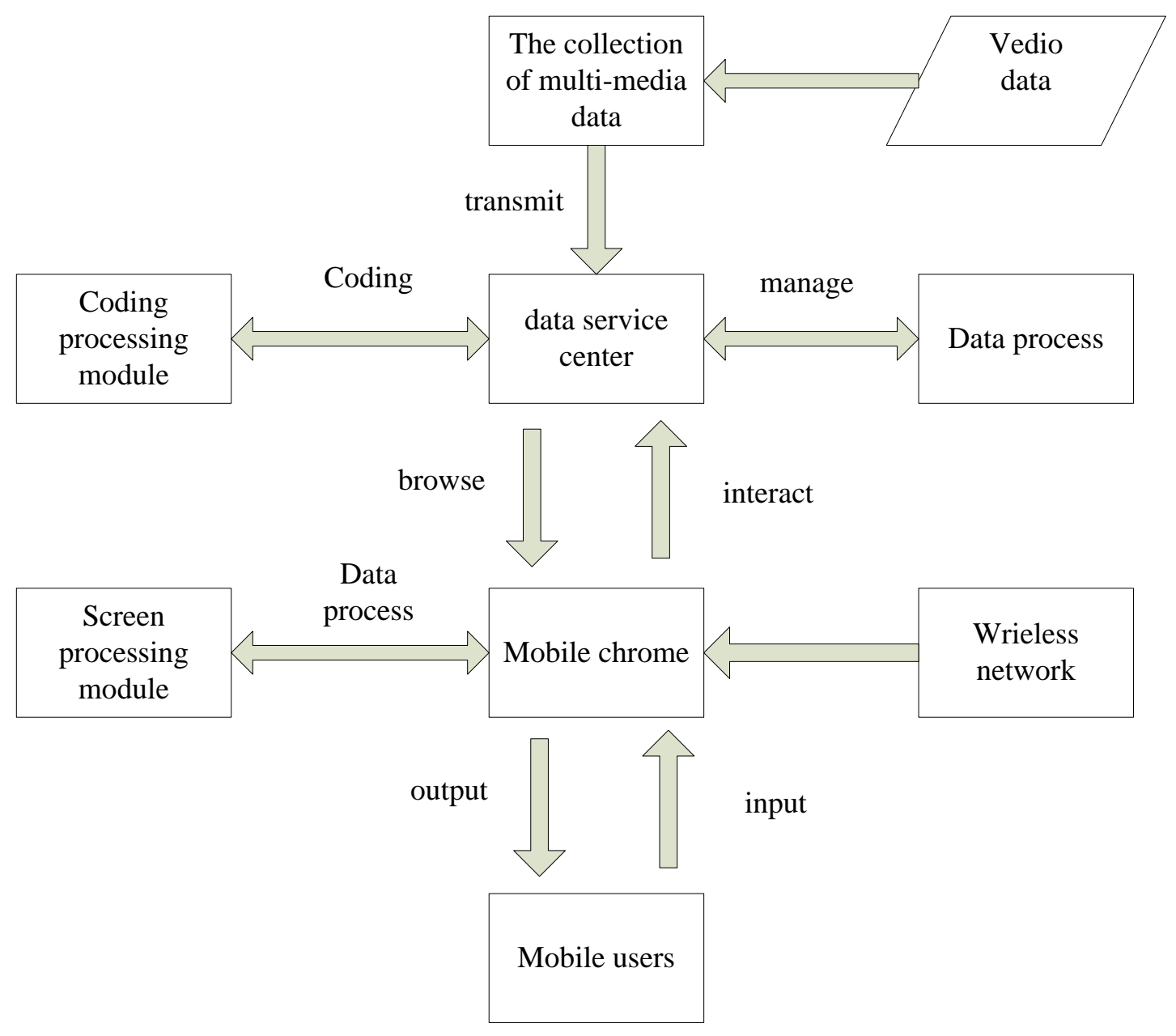

Fig 3 The comparison of the scheme

After RTPPacket is opened, the data generates a new linked node. First compare the timestamp removed with last node timestamp. If it is less than the timestamp of the last node, then compare the timestamp of the penultimate node until timestamp node not greater than that is found.

\section{The display module of terminal screen}

In the process of program calculation, the location of surrounding points should be measured.

Mathematical method for the location of the various points but will increase the complexity, so the method of checking the table is employed in the practice. The location of the corresponding points can decrease of calculation.

\section{Operating environment}

The operating environment of interactive system is: the server is the wireless Laptop and the terminal is simulation PDA developed based on Windows environment. Their communication is dependent on $802.11 \mathrm{~b}(11 \mathrm{Mbps})$. In the following is the Configuration Parameters of PDA

\begin{tabular}{|c|l|}
\hline Operating system & Pocket PC Mobile 2003 \\
\hline CPU & ARMV4 \\
\hline RAM & $64 \mathrm{M}$ \\
\hline resolution ratio & $320 * 240$ \\
\hline Color bits & 16 bits 65536 colors \\
\hline Audio input & Built-in \\
\hline Network & $802.11 \mathrm{~b}$ \\
\hline
\end{tabular}

Table 1: Configuration Parameters of PDA

\section{Summary}

The significance of the research lies in the the application of interactive multimedia technology in 
the wireless environment and changes the traditional service system restricted by location and mobile users-centered. This also enables mobile users to be free from the time and space and makes digital mobile campus, interactive entertainment service and assignment system possible.

\section{Acknowledgements}

Yunnan Development Fund (2012FZ191).

\section{Reference}

[1] Liang G. Effect of delay and buffering on litter-free streaming over random VBR channels[J]. Multimedia, IEEE Transactions on, 2008, 10(6): 1128-1141.

[2] Luan T H, Gaa L X, Shen X. Impact of network dynamics on user's video quality: analytical framework and provision[J]. Multimedia, IEEE Transactions on, 2010, 12(1):64-78.

[3] Parandeh Gheibi A, M. dard M, Odaglar A, et aI. Avoiding interruptions-a qoe reliability function for streaming media applications[J]. Selected Areas in Communications, IEEE Journal on, 2011, 29(5): 1064-1074.

[4] Uwe Steinmann,David Shearer. Reusing multi-media components: a catalogue implementation[J]. Computer Networks and ISDN Systems,1998,3013:.

[5] Mukaddes Erdem,Ferhat Kadir Pala,Turgay Baş. A Usability Study of an Online Instructional Multi-media Discussion Environment[J]. Procedia - Social and Behavioral Sciences,2013,83:. 